\title{
Measurements of the space and time evolution of the surface charge in a dielectric barrier discharge - Comparisons with results from simulations
}

\author{
F. Gégot ${ }^{\mathrm{a}}$, Th. Callegari ${ }^{\mathrm{a}}$, M. Aillerie ${ }^{\mathrm{b}}$, J.P. Boeuf ${ }^{\mathrm{a}}$ \\ ${ }^{a}$ LAPLACE, Université P. Sabatier, Toulouse III, 31062 Toulouse, France \\ ${ }^{\mathrm{b}}$ LMOPS, UMR CNRS 7132, Université P. Verlaine - Metz and Supelec, 57070 Metz, France \\ thierry.callegari@laplace.univ-tlse.fr
}

\begin{abstract}
In this paper we present space and time resolved measurements of the surface charge on the dielectric surface of a dielectric barrier discharge using an electro-optical method based on the Pockels effect. The experimental measurements are compared with results of a 2D fluid model of the discharge in a low pressure (5 torr) coplanar surface dielectric barrier discharge (geometry similar to that of a plasma display panel cell, but larger dimensions).
\end{abstract}

\section{Introduction}

The behaviour of dielectric barrier discharges (DBD) depends strongly on the charge deposited on the dielectric surface. The description of the space and time evolution of this charge is of great interest to optimise these discharges and better understand certain phenomena such as the transition from homogeneous discharges to filamentary discharges, patterns formation or striation-like emission. The aim of this paper is to compare measurements of the surface charge obtained with the Pockels cell method, with results from fluid simulations. From the end of the years 80 , different numerical codes in $1 \mathrm{D}, 2 \mathrm{D}$ or $3 \mathrm{D}^{1}$ dimensions based on the resolution of the two first moment of the Boltzmann equation were developed. These models have been extensively used to better understand the discharge mechanisms and optimise the luminous efficacy. Since the models are based on a fluid description of charged particle transport, they involve strong assumptions on the electron energy distribution function. Detailed comparisons with experiments are therefore extremely useful. In order to study more easily the plasma evolution experimentally we developed a dielectric barrier discharge cell with a geometry similar to that of a Plasma Display Panel (PDP) cell, but with dimensions about 100 times larger, and operating at a pressure about 100 times lower than a real PDP cell. A specific diagnostic based on the Pockels effect was developed to measure the surface charge on the dielectric. The technique is based on the integration of an electro optic crystal which plays the role of the dielectric layer of the DBD ${ }^{1}$. This technique has been previously used for the study of corona discharge and for the measurement of high electric fields $(1-100 \mathrm{kV} / \mathrm{cm})^{2}$. Nevertheless, after taking some necessary precautions, it can also be used for low electric fields such as in Plasma Display Panels (PDP) $)^{3}$. After a brief description of the experimental set-up and the model, we comment the results of the spatio temporal evolution of the charge in PDP geometry in a Ne$\mathrm{Xe} 5 \%$ mixture at 5 torr. We finally discuss the accuracy of the experimental results and the validity of the numerical code.

\section{Description of the experim ental method}

The experimental set-up is based on the Sénarmont arrangement as described in Fig. 1. The optical set-up consists of a $\mathrm{Bi}_{12} \mathrm{GeO}_{20}$ crystal (BGO) followed by a quarter wave-plate and placed between an input polarizer and an output analyzer. The orientation of the various plates are shown in Fig. 1 with respect to the optical axis of the crystal. An He-Ne laser beam 
at $\lambda=632.8 \mathrm{~nm}$ propagates through the optical arrangement and is collected at the output by a Si photodiode. The crystal with dimensions $100 \times 40 \times 7 \mathrm{~mm}^{3}$ used as a dielectric for the PDP is placed in the discharge chamber filled by a Ne-Xe 5\% gas mixture at a pressure of 5 Torr. Additionally during the electro-optic characterizations of the discharge, an ICCD camera was used to take pictures of light emission. These PDP-like discharges operate in a glow discharge regime and are known to be stable and reproducible in time. The signal collected by the photodiode is averaged over 500 cycles in order to increase the signal to no ise ratio, relatively low for this kind of measurement.

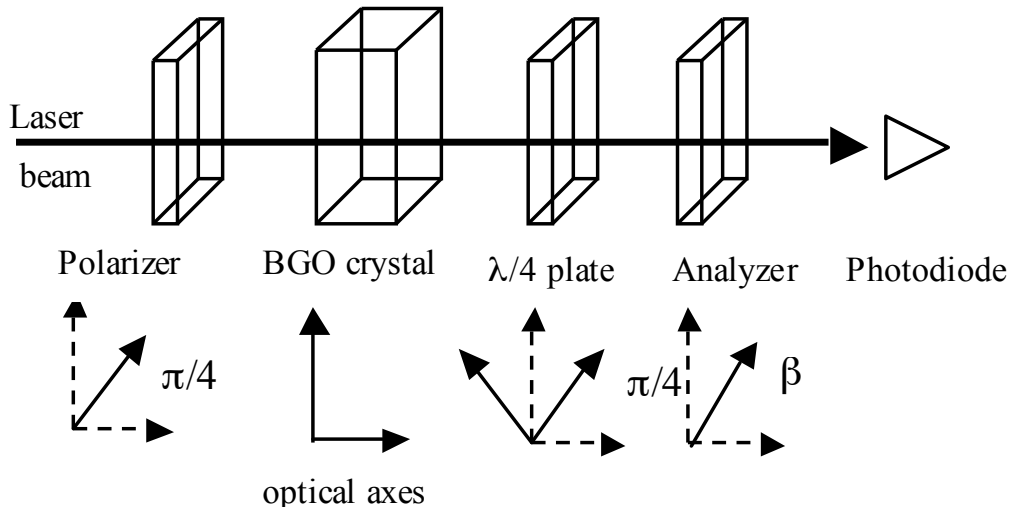

Figure 1: The typical el ectrooptic set-up based on a Sénarmont- type arrang ement

For such systems the transfer function becomes ${ }^{4}$

$$
\mathrm{T}=\mathrm{I}_{0} \mathrm{~T}_{0} \sin ^{2}\left(\frac{\Gamma}{2}-\beta\right)
$$

where $I_{0}$ denotes the intensity of the unpolarized light incident to the input polarizer, $T_{0}$ is the transmission factor of the system and $\beta$ is the azimuthal position of the analyser. As BGO is linearly electro-optic, when an electric field $\mathrm{E}=\mathrm{V} / \mathrm{d}$ (where $\mathrm{V}$ is the applied voltage and $\mathrm{d}$ the electrode spacing) is seen by the crystal, one obtains the expression of the field-induced optical phase retardation,

$$
\Gamma=\frac{2 \pi L}{\lambda} n_{o}^{3} r_{e f f} \frac{V}{d}
$$

$\mathrm{L}$ is the length of the crystal along the beam and $\mathrm{n}_{0}$ is the effective refractive index in absence of applied field. The effective electro-optic coefficient $r_{\text {eff }}$ is linked to the crystal symmetry and to the op to-geometric configuration.

Although the principle of the method looks relatively simple, extreme care must be taken in the characterization of the electro-optic crystal properties to achieve reliable measurements ${ }^{4,5}$.

\section{Description of the model}

The model is identical to the one used in reference ${ }^{1}$. This electrical fluid model consists of the first two moments of Boltzmann equation for electron and ion transport coupled with Poisson's equation for the electric field and with appropriate boundary conditions. The space and time evolution of the electric potential, electron and ion density, and charge distributions on the dielectric layers are calculated. We assume a Cartesian, rectangular geometry of the simulation domain. This implies that the system is supposed to be infinite in the direction perpendicular to the simulation domain, and that the model can yield current per unit length. The model also assumes symmetry bound ary conditions on the sides of the simulation domain perpendicular to the electrodes. On the sides of the domain, which are parallel to the 
electrodes, the potential is imposed at the electrode places, while outside the electrodes the electric field perpendicular to the domain is set to zero.

Poisson's equation is solved at each time step, taking into account the electron and ion densities in the discharge volume, and the charges deposited on the dielectric surface.

\section{Results and discussion}

The DBD geometry is represented in Fig. 2. The voltage is applied on to the crystal

Address electrode

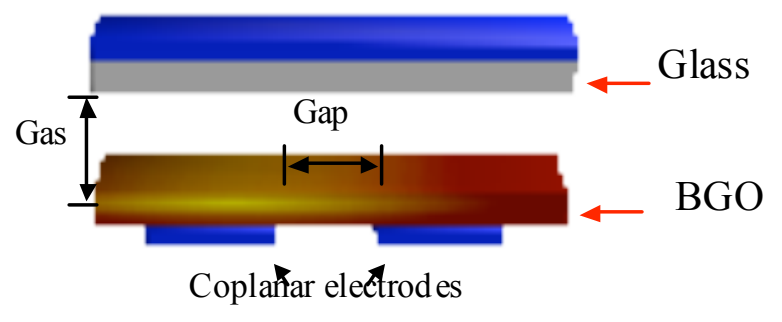

Figure 2 : DBD geometry. through 30 × $30 \mathrm{~mm}^{2}$ coplanar electrodes separated by a gap of $8 \mathrm{~mm}$.

The address electrode is a glass plate of $2 \mathrm{~mm}$ with ITO deposited on. This electrode allows starting the discharge with one of the coplanar electrode. Afterward, it is a floating electrode. As we have seen above in the presentation of the experimental method, one of the dielectric layers is the BGO sample. The second is a glass plate of $2 \mathrm{~mm}$.

At first, see figures 3 and 4, we present and analyze the experimental results of the space and time evolution of the surface charge and the associated current.
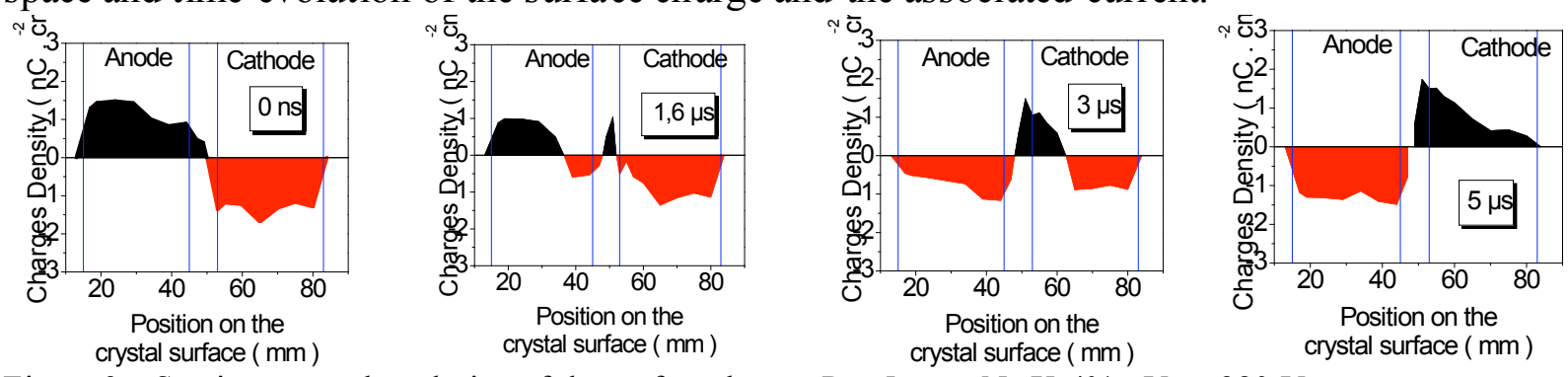

Figure 3 : Spatio temporal evolution of the surface charge. $\mathrm{P}=5$ torr $-\mathrm{Ne}-\mathrm{Xe} 4 \%-\mathrm{V}_{\mathrm{S}}=280 \mathrm{~V}$

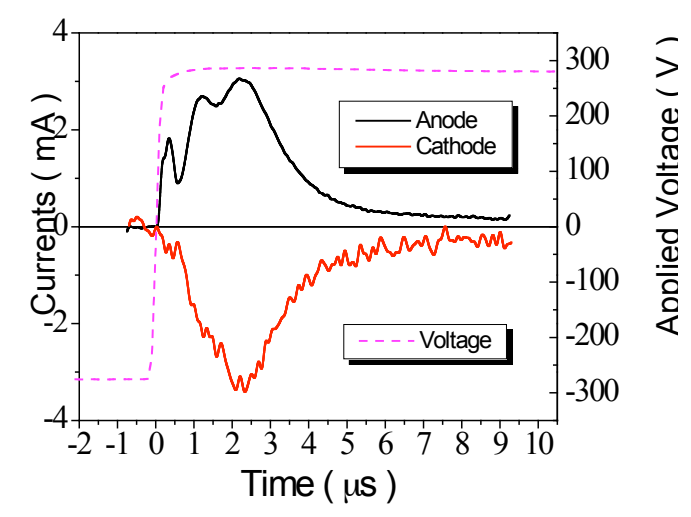

Figure 4: Currents on each electrode and voltage measured in the conditions of figure 3.
The initial distribution of charges is plotted on the left panel of figure 3 with ions in black and electrons in red. The peak density for ions and electrons is around $1.6 \mathrm{nC} . \mathrm{cm}^{-}$ 2 . After the inversion of the polarity on the coplanar electrodes, (see Fig.4) a first current peak is observed around $500 \mathrm{~ns}$ due to the ignition of the discharge between the address electrode and the anode. Electrons start to charge the dielectric surface above the anode near the gap (second panel). Then, the plasma spreads over the two coplanar electrodes until the current reaches a

first maximum at $1.2 \mu \mathrm{s}$. The spreading of the plasma above the anode is faster than the sheath contraction above the cathode due to the higher mobility of electrons (Fig 3-panels 34). A second maximum in the current is observed at $2.2 \mu \mathrm{s}$ and is associated with a second 
maximum of the emission above the cathode. At the end of the current pulse the charge distribution seems to be the mirror of the initial one. The duration of the discharge is $7 \mu \mathrm{s}$.

At second, we present in figure 5 the evolution of the surface charge density calculated with the 2D model with ions represented in black and electrons in red.
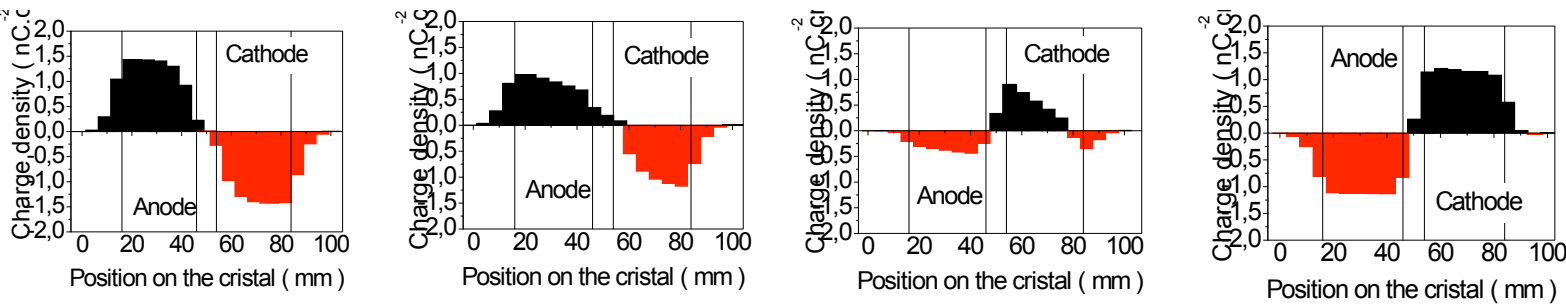

Figure.5 : Spatio temporal evolution of the surface charge density calculated with the 2D model. Conditions are the same than in the experiment. Times are $0,1.6,3$ and $5 \mu$ s from the left to the right panel.

At the beginning the distribution on the two electrodes is uniform with a maximum value about $1.5 \mathrm{nC} / \mathrm{cm}^{2}$. The charges are first deposited above the anode (see Fig. 5-panel 2) just at the edge of the gap. Then the cathode sheath is contracting and sliding along the cathode. Above the anode the electrons are attracting to the edges of the electrode due to the parallel field induce by the potential difference between the former ions and the electrons that are reaching the surface. The final state is reached after $6 \mu \mathrm{s}$ a little bit faster than in the experiment. Another difference between simulation and experience is the absence of breakdown with the address electrode.

From these results, we finally measure and calculate the charge density at the end of the discharge plotted in figure 6. Obtain ed profiles are quite homogeneous on the electrode surfaces. We find that the charge density above the cathode is about $1110^{-9} \mathrm{C}$ and $-11.310^{-9}$ $\mathrm{C}$ above the anode. Simulations performed with the 2D fluid model give similar results.

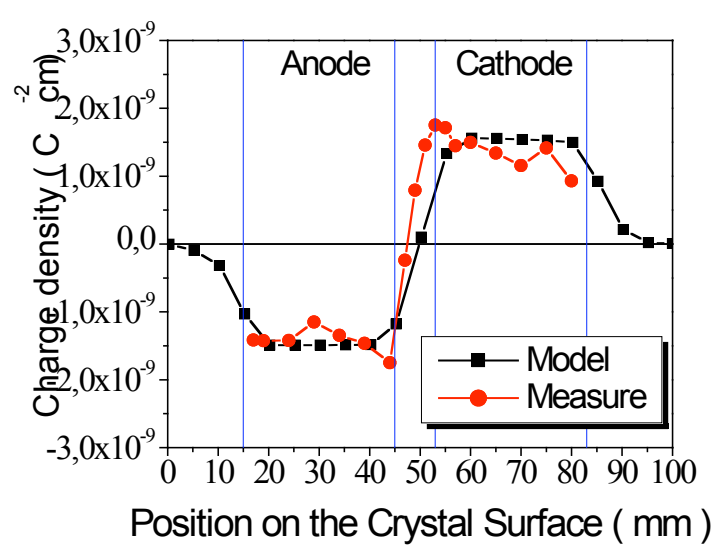

Figure 6: Distribution of the charge density at the end of the period of the signal.
The charge density in this case is about $12,6.10^{-9} \mathrm{C}$ above the cathode and $-12,3 \cdot 10^{-9} \mathrm{C}$ above the anode. For both experimental and numerical point of view, the integration of the current curves gives $+/-1010^{-9} \mathrm{C}$ and $+/-12$ $10^{-9} \mathrm{C}$ respectively. These two results are in good agreement and allow to validate the diagnostic technique and the model.

The results presented in this paper confirm that the model is able to reproduce qualitatively the evolution of the charge at the surface of a dielectric. The difference concerning the breakdown with the address electrode is not due to the description of the charge but could be imputed to the hypothesis used in the model and to the experimental conditions. The quantitative values are also in good agreements but have to be carefully considered. Concerning the experimental method the typical accuracy for our measurements is about $20 \%$. The development of a physical model implies to make some hypotheses but 
also to use input data such as ionisation coefficient, charges mobility or secondary emission coefficient $(\gamma)$. Among these coefficients the more undefined is the gamma. We choose a value of 0.4 for neon gas to get the same firing voltage with the model than in the experiment. The peak value of the current, the duration of the discharge and the charge deposited on the surface depend strongly on this coefficient. Nevertheless, the differences observed between experimental and analytical results are in the range of the uncertainties.

\section{Conclusion}

We have characterized the space and time evolution of the charge at the surface of a dielectric in a low pressure macro cell of plasma display panel with an electro optical method based on the Pockels effect. The measured charges transferred during one current pulse in the considered conditions (DBD in a coplanar electrode geometry at 5 torr in a Neon/Xenon mixture) are between $+/-1.6 \mathrm{nC} . \mathrm{cm}^{-2}$. The time duration of the current pulse is about $7 \mu \mathrm{s}$. The space and time evolution of the surface charge deduced from a $2 \mathrm{D}$ fluid model are in good qualitative and quan titative agreement with the surface charge measurements.

[1] C. Punset, S. Cany, and J.P. Boeuf, J. Appl. Phys. 86, 124 (1999)

[2] A. Kumada, M. Chiba, K. Hidaka, J. Appl. Phys. 84, 3059 (1998)

[3] D.C. Jeong, H.S. Bae, K.W. Whang, J. Appl. Phys. 97, 3304 (2005)

[4] M. Aillerie, N. Theofanous, M.D. Fontana, Appl. Phys. B 70, 317 (2000)

[5] F. Gégot, Th. Callegari, M. Aillerie, J.P. Boeuf, XXVIII ICPIG, July 15-20, Prague, Czech Republic (2007) 